\title{
LETTERS
}

\section{Circulating fibrocytes and pulmonary arterial hypertension}

\section{To the Editors:}

We read with great interest the recent editorial by STENMARK et al. [1] about the potential role of fibrocytes in pulmonary arterial hypertension (PAH) and the original article by NIKAM et al. [2] showing the contribution of bone marrow-derived circulating fibrocytes to hypoxic vascular remodelling and inhibition of their recruitment by treprostinil. Many recent reports indicate a contribution of endothelial and haematopoietic progenitor cells [3], attracted via the CXC chemokine ligand 12/CXC chemokine receptor (CXCR)4 axis in the lung [4], but the role of fibrocytes in PAH is still not clear. A previous study of pulmonary hypertension in rat and calf models has shown that pulmonary adventitial remodelling is due to robust recruitment of nonresident, bone marrow-derived cells that can produce collagen and express smooth muscle cell $\alpha$-actin, thus representing fibrocytes [5]. A unique feature of fibrocytes, described by BuCALA et al. [6], is that they circulate in the bloodstream and are capable of producing extracellular matrix components. They express a variety of mesenchymal markers, including collagen-1, collagen-3, vimentin, haematopoietic markers, such as CD11b and CD45, and the stem cell marker CD34 [6]. Circulating fibrocytes display many functional properties, such as phagocytosis, antigen presentation, cytokine and connective tissue matrix production, and the capacity to proliferate and differentiate. In addition, they respond to a variety of cytokines and growth factors. Fibrocytes also produce angiogenic factors, such as vascular endothelial growth factor and platelet-derived growth factor $\mathrm{A}$, and thus have a potent effect on de novo blood vessel formation, as evidenced by studies in an in vivo Matrigel $^{\mathrm{TM}}$ (BD Biosciences, Franklin Lakes, NJ, USA) model of angiogenesis. Fibrocyte expression of matrix metalloproteinase-9, which mediates endothelial cell invasion, further facilitates the angiogenic process. Fibrocytes have been postulated to influence disease development in a number of situations involving tissue remodelling, such as tumour biology, scleroderma, asthma and pulmonary fibrosis [7].

To extend these studies in human pulmonary diseases, we tested the hypothesis that circulating fibrocytes might contribute to PAH. For that purpose, we quantified circulating fibrocytes in patients with $\mathrm{PAH}$ and examined their possible role as predictors of activity and progression of PAH. Patients were recruited from the French Referral Centre for Pulmonary Hypertension (Université Paris-Sud, INSERM U999, Hôpital Antoine Béclère, Assistance Publique - Hôpitaux de Paris, Clamart, France). All procedures for informed consent, data collection and privacy protection were approved by our institutional ethics committee
(Comité de Protection des Personnes Ile-de-France, Paris VII, France). Diagnosis, based on right-heart catheterisation, demonstrated severe pre-capillary pulmonary hypertension in all cases. Circulating fibrocytes were identified as CD11b+ CD34+ vimentin+ cells, and carefully quantified by flow cytometry after exclusion of doublets and dead cells, among 200,000 events. Preliminary experiments indicated that detection of vimentin was as accurate as collagen I for identifying fibrocytes in triple staining. Fibrocyte number was expressed as an absolute cell count per millilitre of blood and as percentage of total peripheral blood mononuclear cells (PBMCs). In total, 20 patients with idiopathic and heritable $\mathrm{PAH}$ and 18 healthy control subjects were included in this study. The percentage of circulating fibrocytes was decreased in the blood of PAH patients compared with healthy control subjects (mean \pm SEM $0.67 \pm 0.02 \%$ in control subjects versus $0.22 \pm 0.04 \%$ in PAH patients; $\mathrm{p}<0.05$ ) (fig. 1a). However, the number of fibrocytes per millilitre of blood was not significantly different $\left(31,709 \pm 6,444 \mathrm{cells} \cdot \mathrm{mL}^{-1}\right.$ in control subjects versus $16,781 \pm 3,256$ cells $\cdot \mathrm{mL}^{-1}$ in $\mathrm{PAH}$ patients; $\mathrm{p}=0.08$ ) (data not shown). Hence, we proposed that any differences between controls and patients might not rely on circulating number of fibrocytes but on their function. We investigated membrane CD11b expression on circulating fibrocytes as well as on other PBMC populations. We showed an increased expression of $\mathrm{CD} 11 \mathrm{~b}$ on the circulating fibrocytes from $\mathrm{PAH}$ patients $(1,075 \pm 116$ in control subjects versus $2,069 \pm 264$ in PAH patients; $\mathrm{p}<0.001$ ), normalised to the mean fluorescence intensity of $\mathrm{CD} 11 \mathrm{~b}$ of isotype controls, indicating increased activation (fig. 1b-d). We also found CD11b membrane overexpression on PAH monocytes $(1,462 \pm 169$ in control subjects versus $2,425 \pm 278$ in $\mathrm{PAH}$ patients; $\mathrm{p}<0.001)$. Interestingly, monocytes are considered by some authors as progenitors of fibrocytes. The changes in fibrocyte number and activation might be due to an imbalance of some circulating factors acting on fibrocyte differentiation in plasma. For example, it was demonstrated that circulating fibrocytes are sensitive to the cytokine and growth factor environment, as well as to serum amyloid P levels, which have an inhibitory action on their differentiation. It was also demonstrated that addition of the profibrotic cytokines interleukin (IL)-4 and IL-13 to human PBMCs cultured in serum-free medium promotes fibrocyte differentiation, whereas the antifibrotic cytokines interferon- $\gamma$ and IL-12 have inhibitory effects. In further studies, it will be interesting to examine the level of these factors in the plasma of PAH patients. To further explore the functional competence of circulating fibrocytes, we analysed their capacity to differentiate in vitro. We cultured PBMCs in serum-free media for 4 days as previously described [8]. After 4 days, PBMCs were 

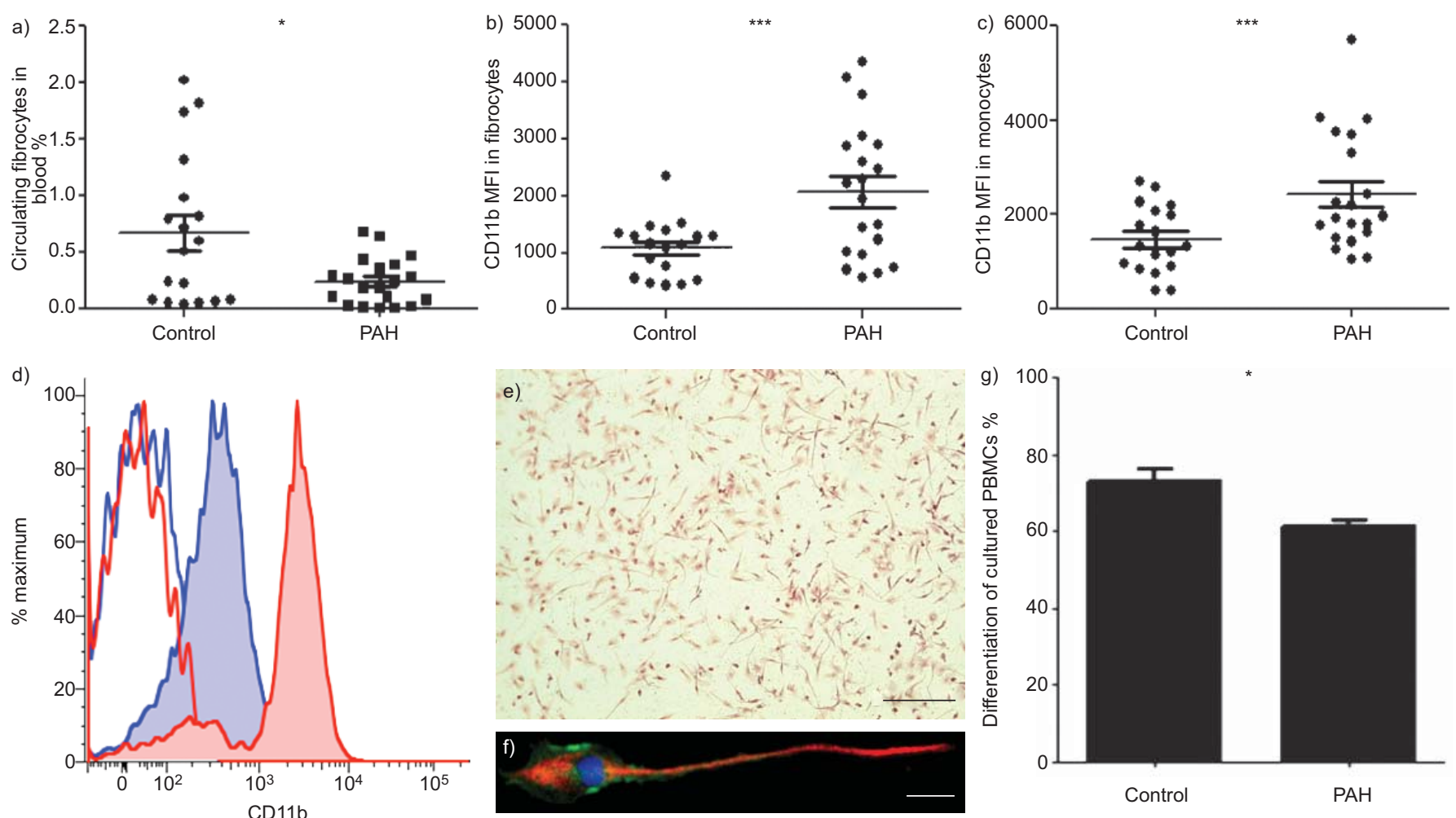

FIGURE 1. Ex vivo and in vitro analysis of circulating fibrocytes in controls compared to pulmonary arterial hypertension (PAH) patients. a) Circulating fibrocyte count in peripheral blood of healthy control subjects and PAH patients, characterised by allophycocyanin (APC)-Cy7-conjugated anti-CD11b and phycoerythrin (PE)-Cy5-conjugated anti-CD34 surface antibodies (BD Pharmingen, Le Pont de Claix, France), and intracellular staining with PE-conjugated anti-vimentin versus PE-conjugated immunoglobulin G1 isotype control (Abcam, Paris, France). CD11b mean fluorescence intensity (MFI) on the b) fibrocyte and c) monocyte populations in peripheral blood, normalised to isotype controls. $n=18$ in the control group; $n=20$ in PAH group. d) Representative histograms for CD11b expression at the cell surface of the CD34+ vimentin+ circulating fibrocyte populations from healthy controls (blue shaded) and PAH patients (red shaded), as compared with isotype controls (blue red and lines, respectively). Flow cytometry data acquisition was performed on a MACSQuant analyser (Miltenyi, Paris, France) and data were analysed using FlowJo software (Tree Star Inc., Ashland, OR, USA). e) Differentiated fibrocytes in culture (haematoxylin and erythrosin staining). f) Immunofluorescent staining for CD45 (green) and vimentin (red). g) Quantification of fibrocyte differentiation in vitro, using antibodies against vimentin (Abcam) and CD45 (BD Pharmingen). Fibrocytes from duplicate wells defined as double-positive, adherent, spindle-shaped cells with an oval nucleus were counted in five different fields per well. $n=4$ in PAH and control groups. PBMC: peripheral blood mononuclear cell. Data are presented as mean \pm SEM. Statistical analysis was performed with GraphPad Prism 5.0 (GraphPad Software, La Jolla, CA, USA), using paired t-test and Mann-Whitney U-test. $*: p<0.05 ; * * *: p<0.001$.

air dried, acetone-fixed and stained with specific antibodies for fibrocyte quantification (fig. 1e-f). We observed a significant decrease in the percentage of differentiated spindle-shaped CD45+ vimentin+ cells in PAH patients in vitro $(73 \pm 3 \%$ in control subjects versus $61 \pm 2 \%$ in PAH patients; $\mathrm{p}<0.05$ ) (fig. $1 \mathrm{~g}$ ). In accordance with NIKAM et al. [2], who found rare fibrocytes in only two cases out of 10, both with plexiform lesions, we did not observe fibrocyte accumulation (i.e. cells expressing both CD45 and vimentin) in the perivascular areas of remodelled small pulmonary arteries in the PAH lungs of four samples we analysed (data not shown). Lastly, we did not find any association between the level of circulating fibrocytes and clinical parameters (pulmonary haemodynamics, New York Heart Association class, 6-min walk distance and plasma brain natriuretic peptide), questioning their overall predictive value in $\mathrm{PAH}$ (data not shown).

From this and previous studies of different aspects of fibrocyte biology in PAH, we can conclude that the contribution of circulating fibrocytes in PAH remains a subject of debate. We have shown a slight decrease in the percentage of circulating fibrocytes in patients with idiopathic and heritable $\mathrm{PAH}$ compared with healthy controls, together with a lower capacity to differentiate in vitro. Low circulating fibrocyte level might be secondary to defects in cell mobilisation mechanisms. To prove this hypothesis, additional studies on the expression of adhesion molecules, such as very late antigen- 4 , CC chemokine receptor 5 and CXCR4, on circulating fibrocytes are needed. Alternatively, it might be due to the low proliferation of CD34+ cells, as recently found in patients with chronic ischaemic heart disease [9], which occurred in the absence of obvious mobilisation defects. Another nonexclusive hypothesis is that the low level of circulating fibrocytes is due to their increased pulmonary vascular recruitment. This hypothesis, raised by FRID et al. [5], is not consistent with data obtained in PAH lungs here and by others [2] describing normal fibrocyte cell count in the perivascular areas of small pulmonary arteries. Furthermore, it remains difficult to extrapolate from studies in the hypoxic model of PAH to the human disease, in which lungs are examined at the pulmonary transplantation time-point, after 
long disease duration. The other important finding of this study is the increased expression of membrane CD11b in fibrocytes. The integrin CD11b (also known as complement receptor type 3, macrophage antigen- 1 and $\alpha_{M} \beta_{2}$ ), which is primarily expressed on neutrophils, macrophages and monocytes, is critical for the normal function of these cells and plays an important role in the development of inflammatory responses. CD11b is expressed in a low-affinity, inactive conformation in circulating leukocytes, but is rapidly upregulated on the cell surface during stimulusdependent activation [10]. The factors triggering upregulation of $\mathrm{CD} 11 \mathrm{~b}$ in fibrocytes and its biological significance are not known. One hypothesis is that the increased expression of $\mathrm{CD} 11 \mathrm{~b}$ in fibrocytes reflects their activation in the course of the inflammatory response, as in patients with $\mathrm{PAH}$. As a consequence of CD11b upregulation, fibrocytes might display an increased capability to adhere to endothelial cells, extravasate and subsequently transmigrate into the surrounding interstitial tissue. Fibrocytes therefore can be proposed to play a critical role in the initiation and maintenance of inflammation in $\mathrm{PAH}$, even though they are not detected in the lung tissues. A central question that remains to be answered is whether fibrocytes differentiate into myofibroblasts, or whether they transiently stimulate differentiation and proliferation of other progenitor cells or resident mesenchymal cells within the lung.

In conclusion, our data suggest that the level of circulating fibrocytes may not be predictive of disease activity and progression, and more detailed analysis of their functional capacity and activation is needed. CD11b upregulation by circulating fibrocytes may have a role in the initiation and/or progression of $\mathrm{PAH}$ but detailed investigations on fibrocyte function will be required.

N. Gambaryan, S. Cohen-Kaminsky, D. Montani, B. Girerd, A. Huertas, A. Seferian, M. Humbert and F. Perros

Faculté de Médecine, Université Paris-Sud, Kremlin-Bicêtre, France. Centre de Référence de l'Hypertension Pulmonaire Sévère, Service de Pneumologie et Réanimation Respiratoire, Hôpital Antoine Béclère, Assistance Publique - Hôpitaux de Paris, Clamart, France. INSERM U999, Hypertension Artérielle Pulmonaire, Physiopathologie et Innovation Thérapeutique, Centre Chirurgical Marie Lannelongue, Le Plessis-Robinson, France. Département de la Recherche Médicale, Centre Chirurgical Marie Lannelongue, Le Plessis-Robinson, France.
Correspondence: F. Perros, INSERM U999, Centre Chirurgical Marie Lannelongue, 133 avenue de la Resistance, 92350 Le Plessis-Robinson, France. E-mail: frederic.perros@gmail.com

Support Statement: This study was supported by HTAP France.

Statement of Interest: Statements of interest for D. Montani and M. Humbert can be found at www.erj.ersjournals.com/site/ misc/statements.xhtml

\section{REFERENCES}

1 Stenmark KR, Frid MG, Yeager ME. Fibrocytes: potential new therapeutic targets for pulmonary hypertension? Eur Respir J 2010; 36: 1232-1235.

2 Nikam VS, Schermuly RT, Dumitrascu R, et al. Treprostinil inhibits the recruitment of bone marrow-derived circulating fibrocytes in chronic hypoxic pulmonary hypertension. Eur Respir J 2010; 36: 1302-1314.

3 Montani D, Perros F, Gambaryan N, et al. C-kit positive cells accumulate in remodeled vessels of idiopathic pulmonary arterial hypertension. Am J Respir Crit Care Med 2011; 184: 116-123.

4 Gambaryan N, Perros F, Montani D, et al. Targeting of c-kit+ haematopoietic progenitor cells prevents hypoxic pulmonary hypertension. Eur Respir J 2011; 37: 1392-1399.

5 Frid MG, Brunetti JA, Burke DL, et al. Hypoxia-induced pulmonary vascular remodeling requires recruitment of circulating mesenchymal precursors of a monocyte/macrophage lineage. Am I Pathol 2006; 168: 659-669.

6 Bucala R, Spiegel L, Chesney J, et al. Circulating fibrocytes define a new leukocyte subpopulation that mediates tissue repair. Mol Med 1994; 1: 71-81.

7 Strieter RM, Keeley EC, Hughes MA, et al. The role of circulating mesenchymal progenitor cells (fibrocytes) in the pathogenesis of pulmonary fibrosis. J Leukoc Biol 2009; 86: 1111-1118.

8 Pilling D, Vakil V, Gomer RH. Improved serum-free culture conditions for the differentiation of human and murine fibrocytes. J Immunol Methods 2009; 351: 62-70.

9 Heeschen C, Lehmann R, Honold J, et al. Profoundly reduced neovascularization capacity of bone marrow mononuclear cells derived from patients with chronic ischemic heart disease. Circulation 2004; 109: 1615-1622.

10 Hogg N, Leitinger B. Shape and shift changes related to the function of leukocyte integrins LFA-1 and Mac-1. J Leukoc Biol 2001; 69: 893-898.

\section{Artificial lung as an alternative to mechanical ventilation in COPD exacerbation}

\section{To the Editors:}

Acute exacerbation of chronic obstructive pulmonary disease (COPD) is commonly treated with different kinds of noninvasive positive pressure devices, ranging from helmet or face-mask continuous positive airway pressure (CPAP) to noninvasive pressure support ventilation (NPPV), or Bi-PAP [1].
The use of positive end-expiratory pressure (PEEP) and NPPV often results in the successful treatment of COPD patients with respiratory distress [1, 2]. If, despite maximal medical management, respiratory distress and gas exchange deteriorate with increasing tachypnoea and acidosis, and with altered level of consciousness, then tracheal intubation and mechanical 